LA-UR -88-1446

$$
\text { CONF-880614 -.2 }
$$

LA-UR--88-1446

DE88 010910

LOS Alamos Natinal Laboratory is Operated by the University of Californis for the United States Deparment of Energy under contract W.7405-ENG-36

TITLE:

\title{
SOURCES OF POLARIZED IONS AND ATOMS
}

AUthor(S): $\quad$ W. D. Cornelius $\quad \therefore ., \%, 1988$

SUBMITTED TO:

7th International Conforence on ion Implentation Tochnology, ITT'88

June 7-10, 1988, Tokyo, Japan

\section{DISCLAIMER}

\begin{abstract}
This report was propured as an eccount of work aponeored by an ugency of the United Sitates Guvernment. Neither the Unised States Covernment nor any ageniy thereof, nor any of their employeses, makes any warranty, expreas of implied, of auumes any legal liability or recponal. bility for the securacy, completeneas, of usofuinews of any informulion, appuratus, product, or proc in discloeed, of represente thet its use would not infringe privately uwned rights. Refoe. ence herein to any apecific cummercial picuduct, proceas, or arvica by irade name. Irademark. manufucturer, or otherwice doen not necesearlly conatitute or imply its endorement, rocom. mendation, of favoring by the United State Clovernment of any agency thereof. The viowt and opinione of authors expreased herein do not necasearilly stule of reflect thoes of the United States Government or any usency thercof.
\end{abstract}

Oy acceptence of this article, the publisher recognizes that the U.S. Government retains e nonexclusive, royalty-free license to publish or reproduce the published form of this contribution or to allow uthers to do so tor U.S. Government purposes

The Les Alames Napisnal Labor ofory requests that the publisher identify this enicle ces work pertormed under the eusplest of the U.S. Oepertment of Energy

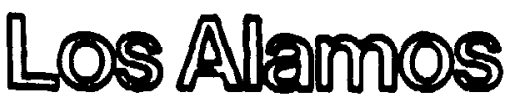

Los Amos Nwitiond Lecrotory

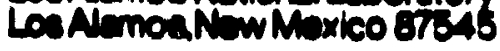

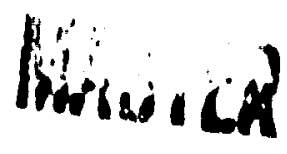




\title{
SOURCES OF POLARIZED IONS AND ATOMS*
}

\author{
Wayne D. Cornelius \\ Accelerator Technology Division \\ Los Alamos National Laboratory, Los Alamos, NM 87545 USA
}

\section{ABstract}

In this presentation we discuss methods of producing large quantities of polarized atoms and ions (Stern-Gerlach separation, optical pumping, and spin-exchange) as well as experimental methods of measuring the degree of polarization of utomic syatems. The usefulness of polarized atoms in probing the microscopic magnetic surface properties of materials will also be discussed.

\section{INTRODUCTION}

Polarized ions have been available from polarized ion sources for nearly thirty years and heve been used primarily in the examination of the spin-dependent terms in the nuclear interaction. It is only more recently that we have begun to apply polarized probes to the elucidation of microscopic electric and magnetic fielde of materials' surfaces $[1,2]$. The concents invoived in the production of polarized probes are relativaly aimple. Howover, as we shall see, the production of intense polarized encembles of atome and ions is a complex process.

Because the nuclear magnetic momente are amall, it is impractical to produce polarised nuclei directly. All of the practical techniquen rely upon the hyperfine coupling betiveen the olectronic rasegnetic momonts of unpalred atomic elertrons and the nuclear magnetic moment. Hence, all sources of nuclear polerization have their roots in atomic phyaics. In what follows, I will diccusa mainly the production of

\footnotetext{
-Work supporlad by the L, S Department of Energy, OMce of High Energy and Nuclear Phyalcs.
} 
polarized hydrogen ions because these sources make up the great majority of operational sources today. However, whenever the techniques can be applied to other atomic species, I will briefly cover that application as ve!l.

\section{THE LAMB-SHIFT SOURCE}

The first practical polarized ion source utilized the Lamb-shift in the $n=2$ levels of atomic hydrogen and its isotopes[3]. Figure 1 shows a Breit-Rabi diagram of the $\mathrm{n}=2$ levols of atomic hydrogen. The Lamb-shift, indicated in the figure, breaks the degeneracy of the $n=2$ levels as shown leaving the metastable $2 S$ level with slightly, higher energy than the 2P-1/2 level and somewhat lower energy than the 2P-3/2 level. As the magnetic field is increased, the energy levels shift as shown in the figure. Those levels with electronic spins aligned with the magnetic field increase in energy with increasing magnetic field. Those with antialigned electronic spins decrease in energy. At about 575 gause, the lower $2 S$ levels (historicall's called the beta states) cross the upper 2P-1/2 levels. Addition of a small electric fiald $(1-10 \mathrm{~V} / \mathrm{cm})$ effectively mixes these levels and the beta states quickly transit to the ground state, leaving only the upper levels occupied (historically the alpha states). In the Lamb-shift source, the metastable beam is formed by a charge-capture reaction of protons with cesium vapor. Approximately one-third of the interacting protons aro formed in the $2 S$ levels. After the quenching of the beta states described above, the resulting atomic beam is passed into a cell containing argon gas. A nearresonance between atomic and ionic energy levels preferentially ionizes the $n=2$ atoms to negative hydrogen ions. The ground-state atoms are only reakly ionized. Hence, the negative ion beam produced will have essentially the same nuclear polarization as the metastable atomic beam.

Following the 675-gaus fleld crosaing and quenching 0 the beta states, the alpha states are atomically polarized (possess a net electronis spin orientation) but possess no net nuclear polarization. Therefore, before tho argen cell, we must tranofurm the atomic polarization into nuclear polarization. 'This is accomplished using a nonediabatic reveral of the megnotic field(4). Eacentially, a "Sonatransition," as this technique is now called, utilizes a reveral of the magnutic fleld direction, which Is rapid with renpect to the Lermor preceseion rate of the atoms. Figure $2 \mathrm{a}$ is a BioitRabi diagram that demonstrates the datails of the Sone-transition. At firat we have oaly the $F=1$ states with orientations of $M F=+1$ and $M F=0$, respectively (left side of tho Fig. 2a). If the magnetic flold is decreased rapidly to vory low values (around 1 
Gauss), the $a_{1}$ state cannot precess fast enough to follow the field. Therefor $\mathrm{e}$, when the magnetic field is subsequently reversed, the atomic spin remains fixed in a direction that is now opposite the applied magnetic field. This is equivalent to a transition from the $a_{1}$ state to the $\beta_{1}$ state. The $a_{2}$ state has $M F=0$ and, thus, no preferred orientation along the magnetic field direction. Therefore, when the magnetic field reverses direction, the a state simply turns itself inside out and continues to be an a state.

If the magnetic field is again increased sufficiently above the critical field (where the nuclear coupling to the external field is equal to the hyperfine coupling), both nuclear spins will be antialigned with the external magnetic field and the electronic spins have no net alignment. Hence, the Sona-transition effectively transfers atomic spin orientation into nuclear spin orientation and subsequent ionization will provide a nuclear-polarized ion beam. Similar arguments can be used to demonstrate that if we start with only the beta states, the Son\&-transition will again provide nuclear polarization (Fig. 2b). Transitions from the beta states cannot be used in the Lambshift source but, as we shall see, will play an important role in later polarized ion. source developments. One of the fundamental limitations of the Lamb-shift source is the rather low ion intensities available. Nature, it seems, has conspired to limit the ion currents available from the Lamb-shift source to approximately one microamr are per ionized hyperfine level (for example, 2 microamperes frcm a source with a Sona-transition) [5].

\section{Atomic-Beam Ground-STATE GOURCE}

To increase the available polarized ion current, the atomic-beam, ground-state cource was developed. In the ground-state source, hydrogen gas is dissociated and formed in to a thermal, superenic atomic beam that is passed through a multipole magnet. The interaction of the atomic magnetic mornent with the magnetic field gradient of the multipole magnet produces a focuning force in those atoms with positive a tomic apin projection (aligned with the magnetic field direction) and a defocusing force in those with negative projections. Hence, aner a short distance, only the atoms with positivespin-projection remain in the beam. This Storn-Gerlach epi ration technique has also been succenfully uned to produce beame of polarized alkall atoms $(6,7]$ and polerized vapor targets [8].

Once again we have produced a beam of a tome polarized in electron spin and unpolarizad in nuclear opin. The electronic polarization can now bo tranaformed into 
nuclear polarization as before. However, the beam velocities involved in the groundstate polarized source are low enough that it is difficult to meet the conditions of a Sona-transition; for example, the rate of change of the magnetic field is large compared with the rate of Larmor precession. In gi und-state sources, nuclear polarization is achieved using rf transitions. By choosing the proper magnetic field and $r$ frequency, we can induce Majorana transitions between the hyperfine levels and induce nuclear polarization in the atomic beam.[9] The low velocities of the ground-state atomic beam reduce the longitudinal dimensions required for the $\mathrm{rf}$ transition to a practical size.

Because the ground-state source utilizes only ground-state atoms, selective ionization through a resonance (as in the Lamb-shift source) is not necessary. The original ground-state source ionizer concept used simple electron impact to produce $\mathrm{H}+$ and $\mathrm{D}+$ ions [10]. Subsequent improvements of the ground-state source heve led to polarized ion currents in excess of $400 \mu \mathrm{A}$ of $\mathrm{H} \because$ and $\mathrm{D}+$ : ions [11]. In order to produce negative ions from the ground-state source, two different concepts were developed into working sources. The first, developed by Gruebler et al. at ETH. Honggerberg, ues a conventional positive ion source and produces the negative ions by double charge exchange in sodium vapor [12]. To date this source has produced up to $16 \mu \mathrm{A}$ of polarized $\mathrm{H}$ - ions [11]. The alternative to the duuble charge-exchange cuncept, developed by Haeberli et al. at the Univi rsity of Wisconsin [13] relies on direct ionization to $\mathrm{H}$ - through interaction with a fast ( $40 \mathrm{keV}$ ) atomic cesium beam. This source has produced up to $40 \mu \mathrm{A}$ of polarized $\mathrm{H}$ - beam at th? Brookhaven National Laboratory synchrotron [14].

\section{Optically pumped Sources}

The final type of polarized ion source that I will discuss is the most recent development. With the recent improvements in high-power dye lasers, it has become possibie to polarize a wide variety of atomic species by direct optical pumping [15]. Severa! of the optically pumped pniarized ion source (OPPIS) ideas, both conceptual [16] and implemented into working models $[7,17,18]$, utilize direct optical pumping of the atomic apecies of interest. A variation of the optical pumping theme usen charge exchange in an optically pumped vapor target $[19,20]$.

Figure 3 innwe schematically an OPPIS based on the charge exchange (CX) principle. Briefly, atomic polerizution is achleved by capturing the polarized electron from the poiarized vapor target. The atomic polarization is then 
transformed into nuclear polarization by the Sona transition described earlier. The reversal of the polarization orientation is achieved by a reversal of the sense of the circular polarization of the laser photons and not by any physical change in the configuration of the source or its magnetic fields. As described earlier, the Sona transition is equally effective for initial alpha states or beta states in transforming atomic polarization into nuclear polarization. Optically pumped CX sources are online and/or under development at the Japanese National Laboratory for High Energy Physics in Tuskuba (KEK) [20], the Tri-University Meson Facility (TRIUMF) in Vancouver BC Canada [21], the Los Alamos Meson Physics Facility (LAMPF) in Los Alamos NM, USA [22 ], and the Soviet Institute for Nuclear Research (INR) near Moscow, USSR $[23,24]$. The source at KEK has produced up to $50 \mu \mathrm{A}$ of polarized $\mathrm{H}$ - beam [20] and the INR source has produced up to $1 \mathrm{~mA}$ of polarized $\mathrm{H}+$ beam [24].

More recently, the concept of spin exchange (SPX) has led to the possibility of polarizing atoms by direct spin-exchange with a polarized target rather than by a cx interaction [25]. The SPX concept is identical to the CX source except that no ions or ion beams are involved in the SPX source. Future development of the SPX source depends critically on achieving high polarizations in dense alkali vapor targets. Given the present rate of laser and target technology development, the SPX source should become available in the very near future $[26,27]$.

The optically pumped source has been called the "universal polarized ion source" [15] because, in principle, it could be used to produce a polarized beam of any atomic species with nonzero nuclear apin. Hence, a wide variety of polarized probes can be made available for studying the properties of materials as outlined in Sec. 6.

\section{Meagurement Of Polarizations at low Energies}

Measurement of polarization at low ener gies has always been a problem. Nuclear polarization is easily determined by acceleration of ions up to several $\mathrm{MeV}$ and measuring asymmetries in a nuclear acattering experiment. This technique is most often impractical because a large accelorator fasility is required.

In the type of laboratory experiments most often encountered, wo have two different cot of conditions. We either haye a well-collimated bean of atome or we heve an ensemble of atoms confined in some manner wuch as $c$ vapor cell. These two conditions require different means of measuring polarizations. 
If we have a beam of atoms and that beam of atoms is sufficiently dense, then the polarization of the ensemble can be measured by direct optical fluorescence. By tuning a single-mode laser ( $\Delta \mathrm{f}<10 \mathrm{MHz}$ ) through the absorption line(s) of the atoms, the strength of the fluorescence signal is proportional to the number density of the particular hyperfine state in resonance with the laser. The inherent directivity typical of atomic beams (for example, low transverse velocities) removes most of the Doppler broadening usually present in vapor targets. Therefore, we easily obtain sufficiently high resolution to resolve the different hyperfine states. This technique is particularly useful for atoms with large transition strengths to the ground-state (such as the alkali atoms). In this case, extremely small numbers of atoms are detectable using optical fluorescence [28].

The atomic polarization can also be determined by passing the atomic beam through a Stern-Gerlach magnet as described above [27]. The transmission through the magnet depends on whether the atomic spin projection is aligned or antialigned with the field.

An alternative to direct optical fluorescence was developed by Dreves et al. $[29,30]$. This technique exploits the fact that singly charged alkali ions have electronic configuration with $J=0$ (in the ionic ground state). Capture of an unpaired, unpolarized electron fiom a thin foil often produces neutral atoms in excited atomic levels. The hyperfine interaction transfers some of the nuclear polarization to the unpaired electron. By measuring the polarization of the light emitted by the excited-state atom in transiting to the ground state, the polarization of the nucleus can be inferred. The anai;zing powers of the various excited states are easily calculated assuming LS coupling and folding the time-dependent electronic polarization with the lifetime of the excited state [31]. Schuler et al. have extended this technique into the vacuum ultraviolet to measure the polarization of hydrogen atoms [32].

For an ensemble of atoms contained within a cell, the polarization can be determined in several different ways. The simplest way is to form a hole in the cell and allow some of the atoms to leak out and form an atomic beam, allowing the techniques described above to be used. However, the polarization of the atoms in the cell tan also be determined ire :tly, along with the integrated number denaity, from the Faraday rotation of the plane of linear polarization of a probe laser pasaing through the cell $[33,34,36,36]$. This effect de pends on virtual abeorption and reemisuion of photons near the abeorption line of the atomic aperies in the cell. The difference between the indices of refraction of the circularly polarized components of 
the linearly polarized light leads to a phase shift between those components and, hence, a physical rotation of the plane of linear polarization. By measuring this rotation angle, the polarization as well as the number density of atoms in the cell can be determined.

A final method of determining the polarization of atoms in a cell exploits the properties of the negative hydrogen ion [37]. This ion only exists in one configuration with the electronic spins antialigned. Hence, by passing a polarized atomic hydrogen beam through the cell, the $\mathrm{H}^{-}$current produced in the cell is proportional to the product of the hydrogenic polarization, the electronic polarization of the atoms in the cell, and the cross section for charge pickup from the atomic species in the cell. Alternatively, a fast proton beam can be used, and the $\mathrm{H}^{-}$current is proportional to the square of the polarization of the atoms in the cell and the polarization capture efficiency of the reaction $[37,38]$.

\section{Polarized Surface Physics}

During the 1985 polarization conference in Osaka, D. Fick reported on a scudy of the surface properties of certain refractory metals using polarized lithium and sodium beams [1]. The vector polarization of a ${ }^{6} \mathrm{Li}$ beam was used as a probe of the microscopic magnetic fields of the metallic surfaces under study, and the nuclear quadrupole moment of a ${ }^{23} \mathrm{Na}$ beam was used as a probe of the microscopic surface electric field gradient.

Figure 4 shows a schematic of Fick's experimental arrangement [31]. Polarized atoms were directed onto a hot refractory metal surface where the atoms were ionized on the surface after some residence time, which depended on the temperature of the surface. The polarization of the emitted ions was measured using the beamfoil spectroscopic technique described above. The parameter measured was the depolarization of the incident lithium and sodium atoms and nuclei as a function of the surface temperature, externel magnetic field, and incident polarization type (vector or tensor). Further, by applying of fields at the surfaces under study, he was able to derive not only whe magnitude and sign of the surface electric field gradient from the Stark-eplitting of the nuclear magnetic resonance spectra, but also the asymmetry parameter, $n$, indicating the deviation of the electric field gradient from rotational aymmetry around a normal vector to the surface (Table I) [31] . Fick's experimental technique is restricted to atomic species with low ionization energies 
(the alkali atoms) absorbed on hot $\left(1000^{\circ} \mathrm{C}\right)$ metallic surfaces with high work functions (the refractory metals). However, he does allude to an ongoing experimental program to measure the nuclear polarizations of desorbing atoms using laser-induced fluorescence [39].

More recently, Schmor et al. have used optical pumping to examine the microscopic magnetic surface properties of several materials that make up the containment wa!ls of their sodium vapor target cell [2]. Figure 5 shows a schematic of their experimental apparatus. The sodium vapor is contained within the walls of a tube made of the various materials under test. The polarization of the sodium was monitored using the Faraday rotation method outlined above. By chopping the pump laser ligh , the decay of polarization with time could be determined. By examining the details of how the optically pumped polarization decays with time, they were able to deduce not only polarization relaxation times characteristic of different, surfaces, but also microscopic magnetic field values at the surface (Table II). The TRIUMF experiments demonstrate the usefulness of polarized atoms in deducing surface magnetic fields. For example, macroscopically stainless steel is nonmagnetic. However, the microscopic domains have significant residual magnetic fields close to the surface (Table II). These microscopic fields are very efficient at depolarizing atoms by way of the magnetic dipole-dipole interaction. The copper metallic surface has somewhat reduced surface magnetic field strength. Coating either material with a mixture of methyl-trimethoxysilane and dimethyldimethoxysilane (dry-film) substantially reduces the microscopic magnetic fields evident at the surface (Table $\Pi$ ).

\section{Conclusion}

As we have seen, the production of polarized atoms and ions has been simplified greatly in recent years with the introduction of intense, tunable dye lasers. Optically pumped polarized ion sources (both CX and SPX) should be capable of providing polarized atoms and ions of nearly all atomic species with nonzero nuclear, atomic, or ionic spins. The interaction of these spins with the microscopic electric and magnetic fields of surfaces leads to depolarization of the atoms and ions. By studying the details of the depolarization process, we can gather information about the microscopic eloctric and magnetic fields at the surface of a solid. 


\section{REFERENCES}

[1] D. Fick, Proc. Sixth Int. Symp. on Polarization Phenom. in Nucl. Phys., Osaka, 1985 J. Phys. Soc. Jpn. 55 (1986) Suppl. p. 423-432..

[2] C. D. P. Levy, P. W. Schmor, and W. M. Law, $\left\langle S_{z}>\right.$ Wall Relaxation Measurements of Optically Pumped Sodium Atoms at High Magnetic Field, Journal of Applied Physics, May 1988 (to be published).

[3] T. B. Clegg has written two excellent review articles on the Lamb-shift source: 1) Proc. Conference on Polarized Proton Ion Sources, Ann Arbor, MI, 1981, AIP Conf. Proc. 80 (1982) 21; 2) Proc. Conference on Polarized Proton Ion Sources, Vancouver BC, 1983, AIP Conf. Proc. 117 (1984) 63.

[4] P. G. Sona, Energ. Nucl. (Milan) 14 (1967) 295.

[5] P. Schiemenz, Helvitica Physica Acta, 59 (1986) 620.

[6] P. Eyelhof, B. Bauer, R. Bottger, S. Kossionides, K. -H. Mobius, Z. Moroz, D. Presinger, R. Schuch, E. Steffens, G. Tungate, W. Dreves, I. Koenig, and D. Fick, Fifth International Symposium on Polarization Phenomena in Nuclear Physics, Sante Fe NM, 1985, AIP Conference Proceedings No. 69 (1981), p. 916.

[7] D. Kramer, E. Steffens, and T. Jansch, in Proceedings of the Workshop on Polarized Targets in Storage Rings, R. J. Holt ed., Argonne National Laboratory Report ANJ_-84-50 (1984), p. 155.

[8] W. Gruebler has written an excellent review of atornic beam polaxized target technology in Proceedings of the Workshop on Polarized Targets in Storage Rings, R. J. Holt, ed., Argonne National Laboratory report ANL-84-50 (1984), p. 223.

[9] W. Haeberli, Ann. Rev. Nucl. Sci. 17 (1967)373. 
[10] H. F. Glavish, Third International Symposium on Polarization Phenomena is Nuclear Physics, Madison WI, H. H. Barschall \& W. Haeberli ed., (University of Wisconsin Press, 1971), p. 267.

[11] W. Gruebler, P. A. Schmelzback, D. Singy, and W. Z. Zhang, Helvitica Physica Acta, 59 (1986)568.

[12] P. A. Schmelzbach, W. Gruebler, V. Konig, and B. Jenny, Fifth International Symposium on Polarizetion Phenomena in Nuclear Physics, Sante Fe, NM, 1985, AIP Conference Proceedings No. 69 (1981), p. 899.

[13] W. Haeberli, M. D. Barker, G. Calkey, C. A. Gossett, D. G. Mavis, P. A. Quin, J. Sowinski, and T. Wise, Fifth International Symposium on Polarization Phenomena in Nuclear Physics, Sante Fe, NM, 1985, AIP Conference Proceedings No. 69 (1981), p. 877.

[14] A. Kponou, J. G. Alessi, A. Hershcovitch, T. O. Niinikoski, AIP Conference Proceedings Na. 158 (1987), p. 585.

[15] D. E. Murnick and M. S. Feld, Fifth International Symposium on Polarization Phenomena in Nuclear Physics, Sante Fe, NM, 19uo, AIP Conference Proceeaings Na. 69 (1981), p. 804.

[16] L. W. Anderson and G. A. Nimmo, Phys. Rev. Lett. 42 (1979) 1620.

[17] W. Dreves, W. Broermann, M. Elbel, W, Kamke, D. Fick, and E. Steffens, Fifth International Symposium on Polarization Phenomena in Nuclear Physics, Sante Fe, NM, 1985, AIP Conference Proceedings No.69 . 381). p. 925.

[18] W. Dreves, W. Kamke, W. Broermann, and D. Fick, Z. Phys. A303 (1981) 203.

[19] L. W. Anderson, Nucl. Instr. \& Meth. 167 (1979) 363.

[20] Y. Mori, A. Takagi, K. Ikegami, S. F'ukumoto, A. Ueno, C. D. P. Levy, and P. W. Schmor, ATP Conference Proceedings No. 158 (1987), p. 605. 
[21] M. Law, C. D. P. Levy, M. McDonald, P. W. Schmor, and J. Uegaki, AIP Conference Proceedings No. 158 (1987), p. 610.

[22] R. L. : ork, M. Dulick, W. D. Cornelius, and O. B. VanDyck, in Proc. International Workshop on Hadron Facility Technology, Santa Fe, NM, F'ebruary 1987, H. A. Thiessen, ed., Los Alamo3 Conference report LA-11130. C, Los Alamos National Laboratory, p. 393.

[23] A. N. Zislenski:, S. A. Kokhanovskii, V. M. Lobashev, and V. G. Polushkin, JETP Letters 42 (1985) $\mathrm{B}$.

[24] A. N. Zelenskii, S. A. Kokharovskii, V. M. Lobashev, V. G. Polushkin, and K. N. Vishnevskii, Helvitica Physica Acta, 69 (1986) 681.

[25] D. R. Swenson, D. Tupa, and L. W. Anderson, Helvitica Physica Acta, 69 (1986) 662.

[26] Y. Mori, A. Takagi, K. Ikegami, S. Fukumoto, A. Uenu, C. D. P. Levy, and P. W. Schmor, Nucl. Instr. \& Meth. A (to be published) (KEK preprint 87.77, September 1987).

[27] A. Ueno, K. Ogura, Y. Wakuta, I. Kumabe, K. O-Ohata, Y. Mori, and S. Fukumoto, Nucl. Instr. \& Meth. (to be published), (KEK preprint 87.164, March 1988).

[28] W. M. Fuirbonk, Jr., T. W. Hansck, and A. L. Schallow J. Opt. Soc. Am. 63 (1975) 199.

[29] H. J. Andra, H. J. Plohn, A. Gaupp, R. Frohling, Z. Physik A281 (1977) 15.

[30] W. Dreves, P. Egelhof, K. -H. Mobius, E. Steffens, G. Tungato, K. Bottger, D. Fick, Z. Phyolk A288 (1978) 413.

[31] B. Horn, E. Koch, and D. Fick, Phya. Rev. Lett. 63 (1984) 934. 
[32] K. P. Schuler, C. J. Liu, and T. J. Gay, Helvitica Physica Acta, 69 (1986) 703.

[33] W. D. Cornelius, D. J. Taylor, R. L. York, and E. A. Hinds, Phys. Rev. Lett. 48 (1982) 870.

[34] Y. Mori, K. Ikegami, A. Takagi, S. Fukumoto, and W. D. Corselius, Nucl. Instr. \& Meth. 220 (1984) 264.

[35] Y. Mori, A. Takagi, K. Ikebami, S. Fukumoto, A. Ueno, C. D. P. Levy, and P. W. Bchmor, AIP Conference Proceedings No. 158 (1987), 610.

[36] Z. Wu, M. Kitano, W. Happer, and J. Daniels, Appl. Optics 28 (1986) 4483.

[37] W. D. Cornelius, Workshop on Polarized Targets in Storage Rings, R. J. Holt ed., Argonne National Laboratory report ANL-84-60 (1984), 385.

[38] E .. Hinds, W. D. Cornelius, and R. L. York, Nucl. Instr. \& Rieth. 189 (1981) 599.

[39] U. Memmert, Ph. D. Thesis, Universitat Marburg, 1986. 
Table I. Measured values of the electric field gradient component $V m$ and anymmetry parameter $\mathrm{n}$ (NMR = nuclear magnetic resonance, $\mathrm{NLM}=$ nuclear level mixing, from Ref. 1).

$\begin{array}{ccccccc}\text { Surface } & \text { Nucleus } & \text { Method } & T(K) & \text { Bo(mT) } & \begin{array}{c}\text { Vzz } \\ \left(\mathrm{V} / \mathrm{c}^{2} \mathrm{~m}\right. \\ \left(\times 10^{16}\right)\end{array} & \eta \\ \text { W(110) } & 6 \mathrm{Li} & \text { NMR } & 1130 & 16.3 & 0.0(4) & \ldots \\ & 7 \mathrm{Li} & \text { NMR } & 1210 & 47.9 & -1.1(1) & \ldots \\ & 23 \mathrm{Na} & \text { NMR } & 1290 & 111.6 & -47.4(3) & 0.07(1) \\ & 23 \mathrm{Na} & \text { NLM } & 1360 & \ldots & -49.0(4) & 0.08(2) \\ & & & & & & \\ \text { W-O } & 6 \mathrm{Li} & \text { NMR } & 1160 & 16.3 & +3.08(8) & \ldots \\ & 7 \mathrm{Li} & \text { NMR } & 1265 & 47.9 & +2.59(2) & \ldots \\ & 23 \mathrm{Na} & \text { NMR } & 1245 & 111.6 & -46.8(1) & 0.02(1) \\ & 23 \mathrm{Na} & \text { NLM } & 1360 & \ldots & -49.8(1) & 0.02(1)\end{array}$

Table I. Tabulation of local magnetic nield and correlation time for different wali materials (LMF = local magnetic fleld, from Ref. 2).

$\begin{array}{ccc}\text { Material } & \text { LMF (kG) } & \begin{array}{c}\text { Corrolation Time } \\ \left(\times 10^{12}\right)\end{array} \\ \begin{array}{c}\text { 316 Stainless } \\ \text { otael }\end{array} & 4.01 \pm 0.13 & 6.6 \pm 0.2 \\ \begin{array}{c}\text { Copper } \\ \begin{array}{c}\text { Dry-Film } \\ \text { contod wall }\end{array}\end{array} & 0.14 \pm 0.07 & 19.4 \pm 0.5 \\ & 0.48 \pm 0.02 & 82.6 \pm 2.2\end{array}$


Figure Captions

Fig. 1. Breit-Rabi diagram of the $n=2$ levels in atomic hydrogen. The solid lines in the figure show the relative state energies as a function of the magnetic field. The Lamb-shift is marked on the vertical axis. Each line shown is really a doublet consisting of proton spin-up and proton spin-down states. The lower 25 levels (traditionally callod the beta states) cross with the upper $2 \mathrm{P}$ levels at around 575 Gauss (arrow). In this magnetic field, a small transverse electric field will effectively mix the 25 and $2 P$ levels, causing the immediate ( $<2$ ns) transition of the beta states to the ground state. The upper 25 levels (traditionally called the ulpha states) are unaffected.

Fig. 2. Breit-Rabi diagram of the energies of the hyperfine s-states of hydrogen as a function of the ratio of the external magnetic fleld (B) to the magnetic field produced by the orbiting electron at the position of the nucleus (BC). By scaling in this manner, these diagrams for beth ground-state and metastable hydrogen atoms. Initially the magnetic ficld is oriented along the direction of motion of the atoms (left side of the figures). The magnetic field decreases linearly from left to right, reversing direction in the center.

Figure 2 a demonstrates the Sona transition for initial alphe states (labeled 1 and 2). The dotted lines show the occupled levels and the dashed lines show the unoccupled levels. After the fleld reveral, the atoms occupy only levels 2 and 3. These levels have opposite electronic spin orientotion and equal nuclear orientation. Figure 26 demonstrates the Sona transition for initial bote stotes (labeled 3 and 4). After the fleld reversal, the otoms occupy only levels 1 and 4. These levels also have opposite electronic spins and equal 
nuclear spins. Hence the field reversal has converted electronic polarization into nuclear polarization.

For an efficient Sona transition, the magnetic field should change rapidly with respect to the Larmor precession rate of the atoms in that field. Hence when the field reverses direction. the hyperfine states with zero projection along the magnetic field (states 2 and 4) are unaffected. Those hyperfine states with anguler momentum projection aligned or antialigned with the field (states 1 and 3), cannot precess rapidly enough to follow the field reversal. Hance they remein fixed in space in a direction which is now opposite to the external magnetic field.

Fig. 3. Diegram of the KEK polerized ion source based on optical pumping and charge exchange. The proton beem is produced in an electron-cyclotron resonance (ECK) lon source and pessed into sodium vepor target cell which is optically pumped with a laser. Th.e magnetic field reverses direction between the polarized eodium target cell and an unpolarized sodium target cell used to form negative ions by electron capture (the lonizing cell). This figure is from reference 20.

Fig. 4. Schematic diagram of the experimental apparatus of Horn, Koch, and Fick [31]. The polarized atoms were directed onto a hot rufractory matal surface where they were subsequently ionized atter a mean residence time which depended upon the surface temperature. Following deflection of the lons out of the stomic bsam, the reniaining polerizetion of the stoms was enalyzed the lons using a beam-foil spectroscopic technique described in reference 30. This flgure is from reference 31. 
Fig. 5. Schematic diagram of the experimental apparatus of Levy, Schmor, and Law [2]. The sodium vapor was contained in a target cell with replacible wall materials. The sodium was optically pumped using a laser. The average polarization of the sodium vapor was determined from Faraday rotation $[33,34,35,36]$. This figure is from reference. 2 . 


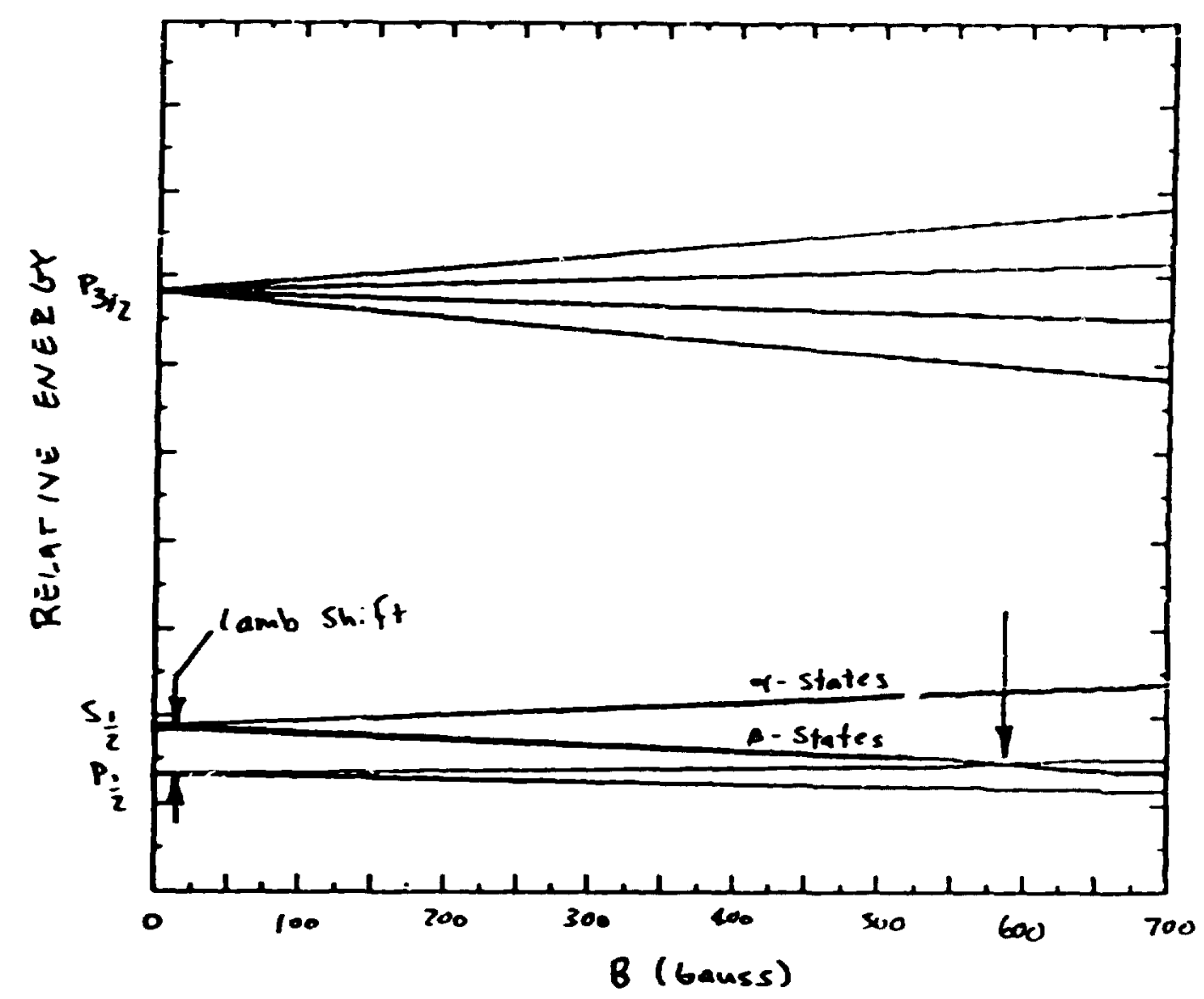

FIg. 1. 


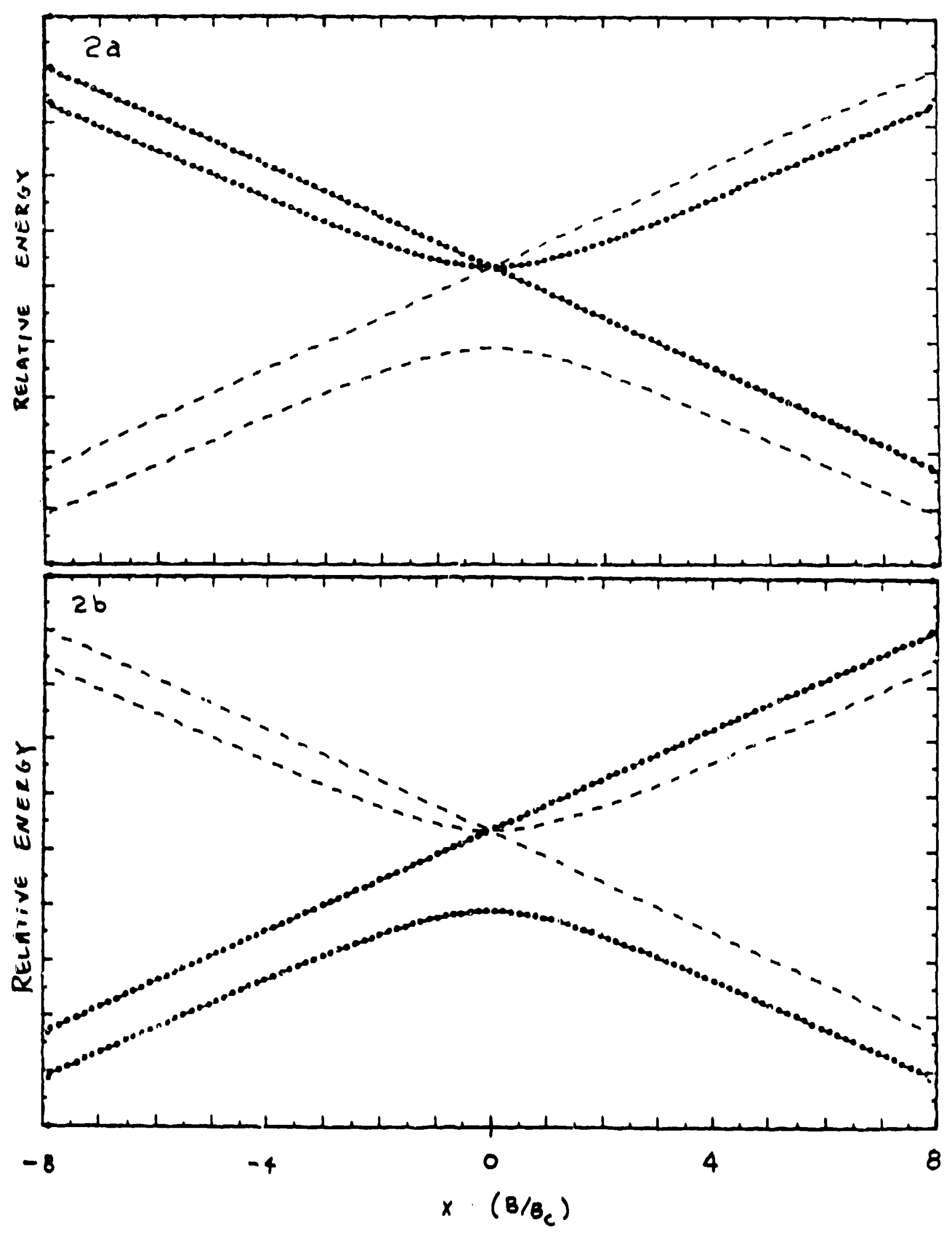

FIG. 2. 


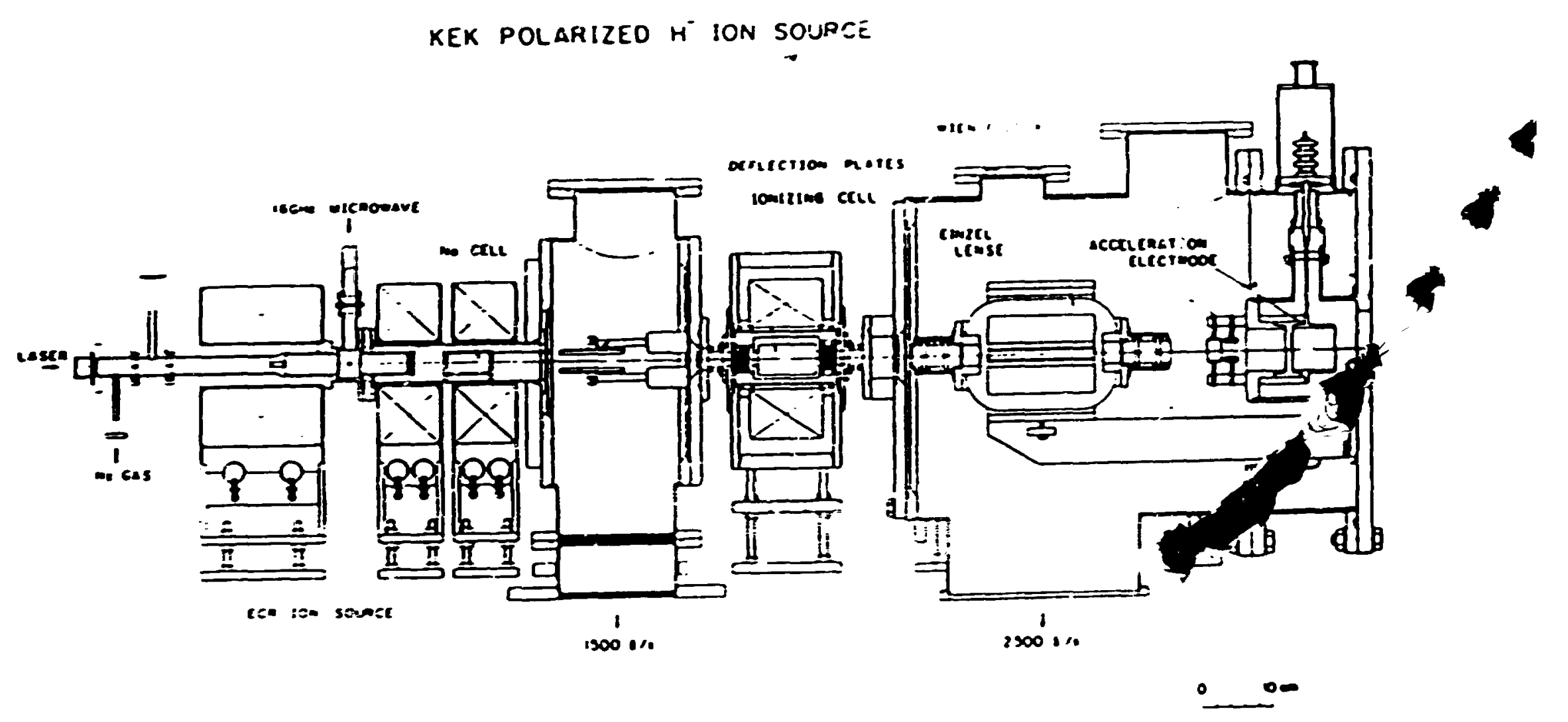

FIG. 3. 


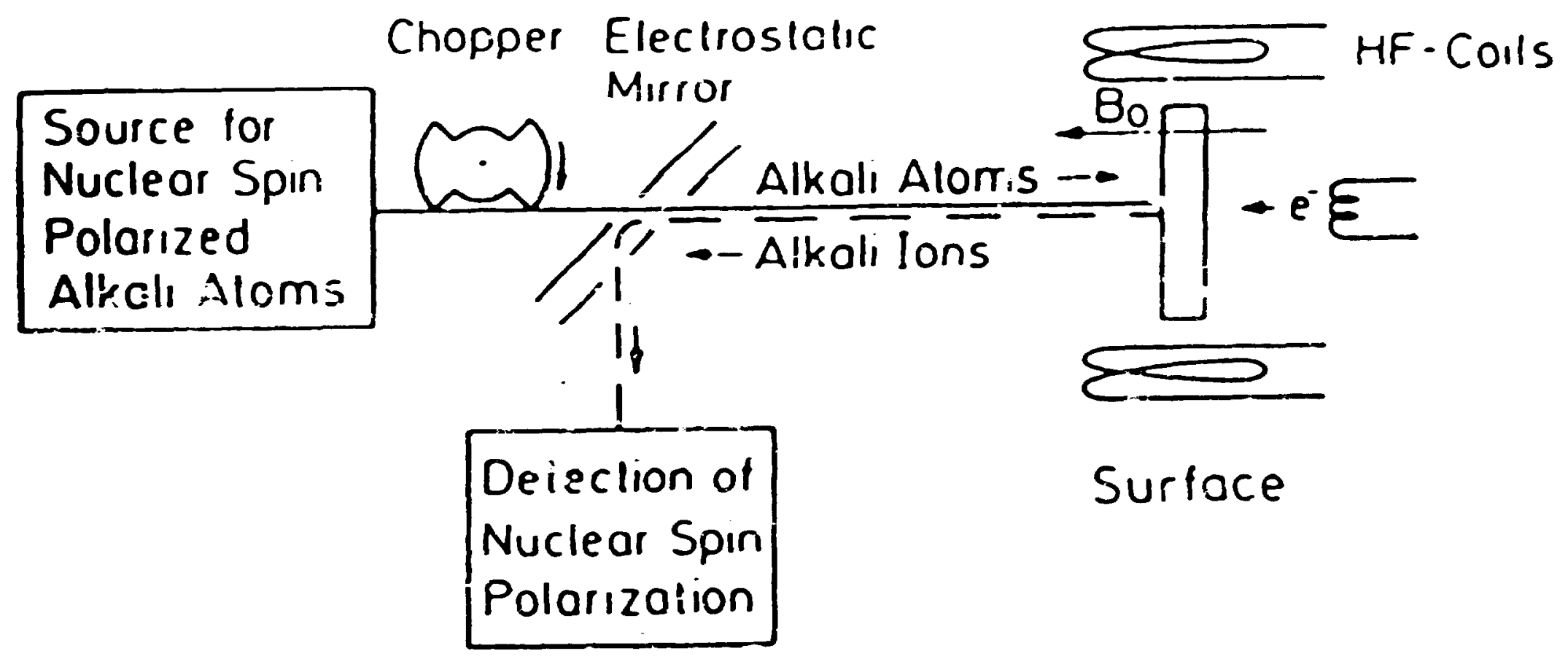

Fi6. 4 


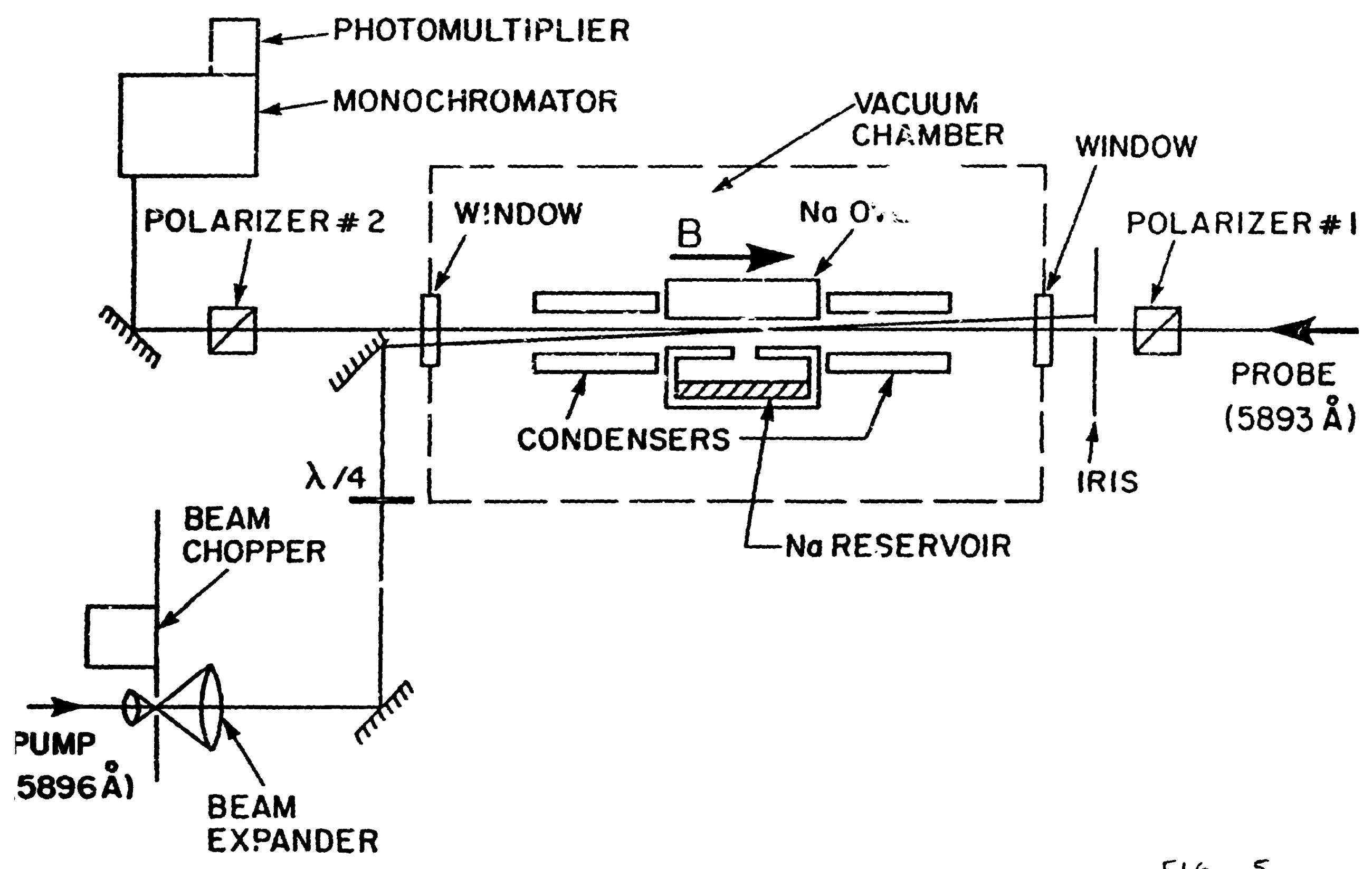

FIG. 5. 